\title{
Automated Data Collection for Serial Section TEM
}

\author{
D. Fellmann, J. Pulokas, B. Carragher and C.S. Potter \\ Department of Cell Biology and National Resource for Automated Molecular \\ Microscopy, The Scripps Research Institute, 10550 North Torrey Pines Road, La Jolla, \\ CA 92037
}

Acquiring high magnification images of a long series of sections is a difficult and labor intensive process. A region of interest must be tracked across the entire set of sections which might be distributed over many specimen grids. This requires examining the sections at a variety of scales prior to acquiring the high magnification images in order to ensure the integrity of the region of interest across the entire set. Multiscale imaging of this sort is not straightforward because the image formed by an electron microscope shifts and rotates as the magnification is changed. The overall task of reconstructing a three dimensional volume from a set of serial sections thus presents a challenging and time consuming process that has limited the number of large scale reconstructions to a few spectacular examples.

We have prototyped a software application to automate data collection for most of the tasks required for serial section imaging. Data is collected during two passes through the grid set using the Leginon software [1]. During the first pass, a low resolution $(75 \mathrm{x}$ magnification) atlas is acquired of each grid (fig. 1) by automatically collecting a mosaic of 17 images. The user then browses through these atlases and identifies the corners of the trapezoidal sections on each grid. Each grid is then imaged a second time to acquire an image mosaic for each section at higher resolution (1500-2000x magnifcation) (fig. 2). A robotic arm is used to load the grids into the microscope [2] for both imaging passes. The grid is removed from the holder after the first pass and must be reloaded for the second pass, which results in small changes in the relative position of the grid between the two passes. Realignment is achieved by collecting a second atlas of the grid at the start of the second pass, determining the shift and rotation between the first and second atlases, and adjusting the targeted trapezoids accordingly.

The image mosaics are automatically aligned and prepared for presentation using the Virtual Microscope (VM) viewer [3], a software application that allows a user to pan, zoom, and step through the collected sections (fig. 3). We have developed a utility that automatically extracts images and metadata from the Leginon database to create the XML and hierarchical image files that are used by the VM viewer. The alignments between the sections and the magnification scales are based on the goniometer (or image shift) coordinates and the relative location of the corners of the trapezoidal section in the atlas. The Virtual Microscope viewer provides the user with the ability to browse and annotate the section data.

We have demonstrated the feasibility for automatically collecting and aligning serial sections for TEM by developing a prototype system that will automatically prepare the data for viewing using an application called the Virtual Microscope viewer. In the future, 
we intend to extend this tool by incorporating a method for automatically identifying the corners of the trapezoidal sections and by providing the user with options for changing the relative order of the sections.

\section{References}

[1] C. Suloway et al., J. Struct. Biol., 151 (2006) 41-60.

[2] C.S. Potter et al., J. Struct Biol., 146 (2004) 431-440.

[3] http://virtual.itg.uiuc.edu

[4] The serial sections were provided to us by Brad Marsh from the University of Queensland. This work was supported by a grant from the National Science Foundation (DBI 0352386).

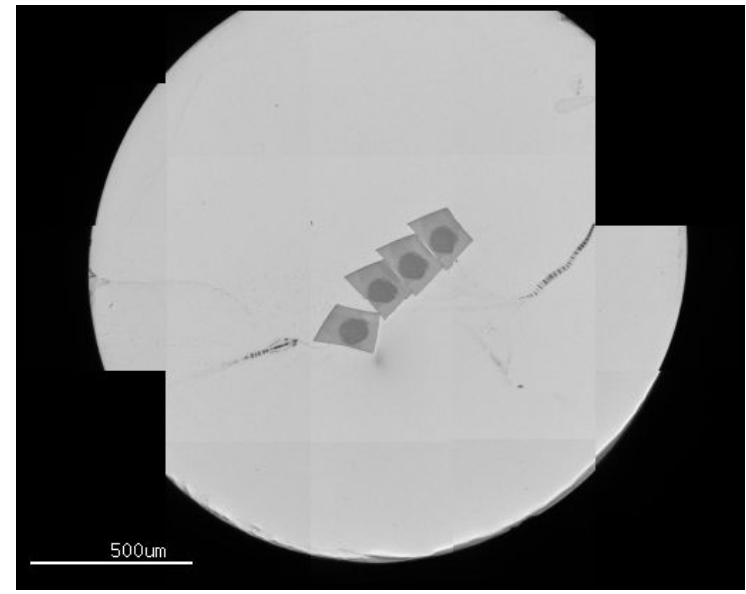

Fig. 1. Whole grid atlas acquired during first imaging pass.

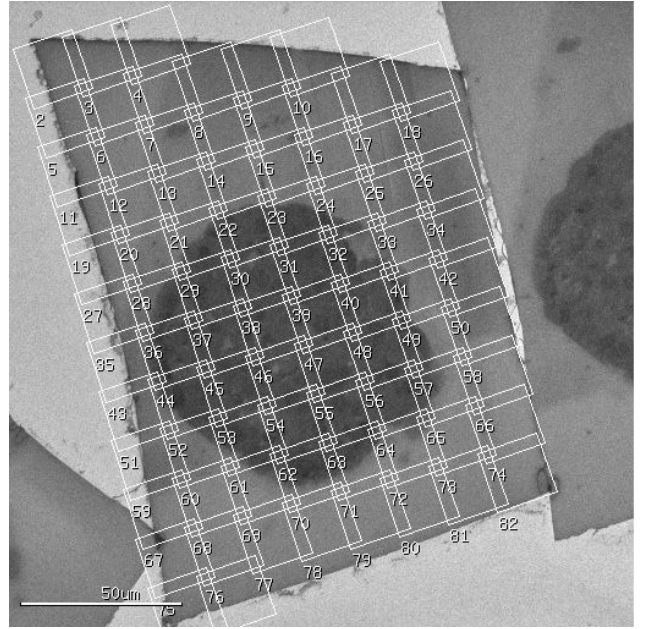

Fig. 2. Targeting of images for second imaging pass.

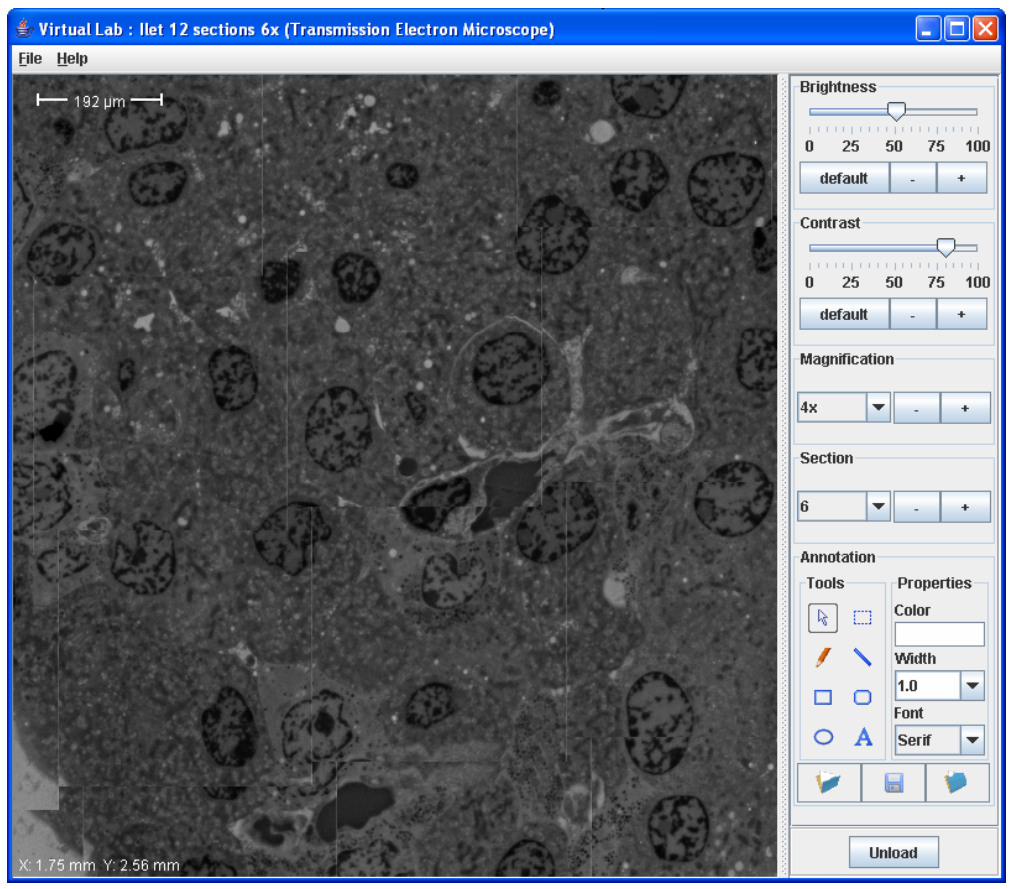

Fig. 3. Virtual Microscope viewer interface that allows the user to step, pan and zoom through the sections. 